\title{
Prognostic Utility of Histological Growth Patterns of Colorectal Lung Oligometastasis
}

Son Jae Yeong · Min Gyoung Pak Hyoun Wook Lee ${ }^{1}$. Seung Yeon $\mathrm{Ha}^{2}$ Mee Sook Roh

Department of Pathology, Dong-A University College of Medicine, Busan; 'Department of Pathology, Samsung Changwon Hospital, Sungkyunkwan University School of Medicine, Changwon; ${ }^{2}$ Department of Pathology, Gachon University of Medicine and Science, Incheon, Korea

Received: August 20, 2017

Revised: December 10, 2017

Accepted: December 27, 2017

\section{Corresponding Author}

Mee Sook Roh, MD, PhD

Department of Pathology, Dong-A University College

of Medicine, 32 Daesingongwon-ro, Seo-gu, Busan 49201, Korea

Tel: +82-51-240-2833

Fax: +82-51-243-7396

E-mail:msroh@dau.ac.kr

\begin{abstract}
Background: Patients with resectable colorectal lung oligometastasis (CLOM) demonstrate a heterogeneous oncological outcome. However, the parameters for predicting tumor aggressiveness have not yet been fully investigated in CLOM. This study was performed to determine the prognostic value of histological growth patterns in patients who underwent surgery for CLOM. Methods: The study included 92 patients who were diagnosed with CLOM among the first resection cases. CLOMs grow according to three histological patterns: aerogenous, pushing, and desmoplastic patterns. The growth patterns were evaluated on archival hematoxylin and eosin-stained tissue sections. Results: The aerogenous pattern was found in $29.4 \%(n=27)$ of patients, the pushing pattern in $34.7 \%(n=32)$, the desmoplastic pattern in $6.5 \%(n=6)$, and a mix of two growth patterns in $29.4 \%(n=27)$. The size of the aerogenous pattern was significantly smaller than that of metastases with other patterns $(p=.033)$. Kaplan-Meier analysis demonstrated that patients showing an aerogenous pattern appeared to have a poorer prognosis, which was calculated from the time of diagnosis of the CLOM $(p=.044)$. The 5 -year survival rate from the diagnosis of colorectal cancer tended to be lower in patients with an aerogenous pattern than in those who had a non-aerogenous pattern; however, the difference was marginally significant $(p=.051)$. In the multivariate Cox analysis, the aerogenous pattern appeared as an independent predictor of poor overall survival (hazard ratio, 3.122; 95\% confidence interval, 1.196 to $8.145 ; p=.020$ ). Conclusions: These results suggest that the growth patterns may play a part as a histology-based prognostic parameter for patients with CLOM.
\end{abstract}

Key Words: Colorectal neoplasms; Lung; Oligometastasis; Growth pattern; Prognosis
The term "oligometastasis" indicates an intermediate state $(\leq 5$ metastases) of malignancy that lies between a localized tumor and widespread metastases. ${ }^{1}$ The implication of the concept of an oligometastatic condition is that metastatic disease may be cured with metastasis-directed, locally ablative therapies. ${ }^{2}$ Colorectal cancer (CRC) is one of the most frequently reported malignant tumors in a surgical series of oligometastasis, ${ }^{2}$ and early metastatic progression of CRC may present with oligometastatic disease. $^{3}$

The lung is the most common extra-abdominal organ of metastases from CRC, involved in 10\%-25\% of all patients with CRC. ${ }^{4,5}$ Evidence suggests that pulmonary metastasectomy is the standard therapy for patients with resectable lesions and has a better prognosis, with a 5-year overall survival rate of $36 \%$ $63 \%$. 4 However, patients who undergo complete surgical resection of these lesions reveal a heterogeneous oncological outcome; some experience recurrent disease and die within a short duration after pulmonary metastasectomy, whereas other patients live without recurrence of disease. ${ }^{4-8}$

In previous studies, many authors have tried to clarify various prognostic parameters in patients undergoing pulmonary metastasectomies, including the number of metastatic lesions, diseasefree interval, pretreatment carcinoembryonic antigen level, and degree of mediastinal lymph node involvement. ${ }^{5,9}$ However, factors for classifying the potential aggressiveness have not yet been fully evaluated in colorectal lung oligometastasis (CLOM). Moreover, little is known about the histopathological features that determine prognosis after pulmonary metastasectomy. ${ }^{10}$

Vermeulen et al. ${ }^{11}$ reported three histological growth patterns with biological differences for colorectal liver metastases: (1) a replacement growth pattern, where tumor cells infiltrate the liver cell plates by replacing the hepatocytes and co-opting hepatic stroma and sinusoids; (2) a pushing growth pattern, where the metastatic tumor expands by compression of the surrounding liver parenchyma with parallel running to the tumor liver interface; and (3) a desmoplastic growth pattern, in which the metastatic 
tumor is separated by a fibrous rim from the liver parenchyma. Although the morphological classification described by Vermeulen et $a l^{11}$ has been reported in a few studies, the prognostic significance remains controversial in patients with colorectal liver metastasis. $^{12-15}$

Furthermore, the significance of similar histological growth patterns has not yet been assessed in patients with CLOM. Therefore, we hypothesized that these histological growth patterns could help predict survival in patients with CLOM. This study was performed to determine the association of these three growth patterns of CLOM with standard clinicopathological parameters and also to determine the prognostic value of these growth patterns in patients who underwent surgery for CLOM.

\section{MATERIALS AND METHODS}

\section{Patients and tissue samples}

This study included 92 consecutive patients who were diagnosed with lung oligometastasis from colorectal adenocarcinoma among the first resection cases. The patients underwent curative lung resection for CLOM between 2004 and 2013 at Dong-A University Medical Center. We excluded patients who had another active cancer, more than five progressive metastatic sites, and a diagnosis of hereditary nonpolyposis CRC syndrome or familial polyposis. In addition, we excluded patients whose metastases showed a positive margin or cautery artifact due to the surgical procedure. ${ }^{12}$ The final sample included 57 men and 35 women, with ages ranging from 31 to 83 years (mean age, 60.8 years). For each patient, the following demographic and clinicopathological factors were collected through a review of medical records: age, sex, differentiation grade of the primary tumor, location of the primary tumor, invasion depth of the primary tumor, lymph node status of the primary tumor, detection timing of CLOM in relation to detection of the primary tumor (synchronous or metachronous), number of CLOMs, diameter of the largest metastasis, and recurrence status. Synchronous lung metastasis had to be diagnosed during the diagnostic work-up or within 3 months following the diagnosis of CRC. CLOMs were defined as metachronous when they occurred at least 3 months after the diagnosis of CRC. ${ }^{7}$ In patients who had undergone multiple resections for metastatic tumors, only the first resection was included in this study. The follow-up of patients began the month following CRC operation and ended at death or completion of follow-up (30 April 2017). All patients signed a written informed consent form to permit the use of their clinicopathological data for the purpose of the study. This retrospective study was approved by the required institutional review boards (IRB No. DAMC 15-141).

\section{Histopathological evaluation}

The growth pattern of CLOM was evaluated on archival $4 \mu \mathrm{m}$ hematoxylin and eosin (H\&E)-stained tissue sections cut from formalin-fixed, paraffin-embedded specimens from the tumorlung boundary. In patients with multiple metastases, sections of the lesion with the largest diameter were examined. The histological growth pattern of CLOM was characterized as the "aerogenoustype," the "pushing-type," or the "desmoplastic-type," according to the modified Vermeulen's procedure. ${ }^{11}$ Briefly, in the aerogenous pattern, tumor clusters, nests, or single tumor cells spread into the air spaces in the lung parenchyma surrounding the edge of the tumor without destruction of the lung architecture and lung parenchyma, which were in close approximation, and showed no compression of the air spaces, desmoplastic stroma or inflammatory infiltrate. In the pushing pattern, the metastasis grew by compression of the lung parenchyma with parallel running to the tumor-lung interface without desmoplastic stroma, and only a mild inflammatory infiltrate. In the desmoplastic pattern, the metastatic tumor was separated from the lung parenchyma by a desmoplastic fibrous rim infiltrated with lymphocytes. There was no direct contact between tumor cells and lung parenchyma. Hence, a "mixed-type" growth pattern was defined as a histologic feature where at least two growth patterns existed, both patterns appearing in at least $20 \%$ of the tumor-lung interface. ${ }^{13}$ Mixedtype metastases were included with a predominant primary growth pattern and one or two secondary growth patterns (Fig. 1). Two pathologists (S.Y.H. and M.S.R.) blinded to the clinicopathological information and outcome evaluated the H\&E-stained slides of metastatic tumor nodules. The third pathologist (H.W.L.), who was also blinded to the clinicopathological information and outcome, resolved any discrepancy between the first two pathologists.

\section{Statistical analysis}

Comparisons between the growth pattern and clinicopathological factors were analyzed using a chi-square test for proportions and analysis of variance (ANOVA) test for continuous variables as appropriate. The patient survival rate was analyzed using the Kaplan-Meier method, and the log-rank test was used to determine if there were any significant differences between the survival curves. All variables that revealed a statistically significant difference on univariate analysis were entered into the multivariate analysis. A p $<.05$ was considered statistically significant. All statistical analyses were performed using the Statistical Package 
for the Social Sciences (SPSS) ver. 21 (IBM Co., Armonk, NY, USA).

\section{RESULTS}

\section{Clinicopathological characteristics}

The clinicopathological characteristics of the study participants
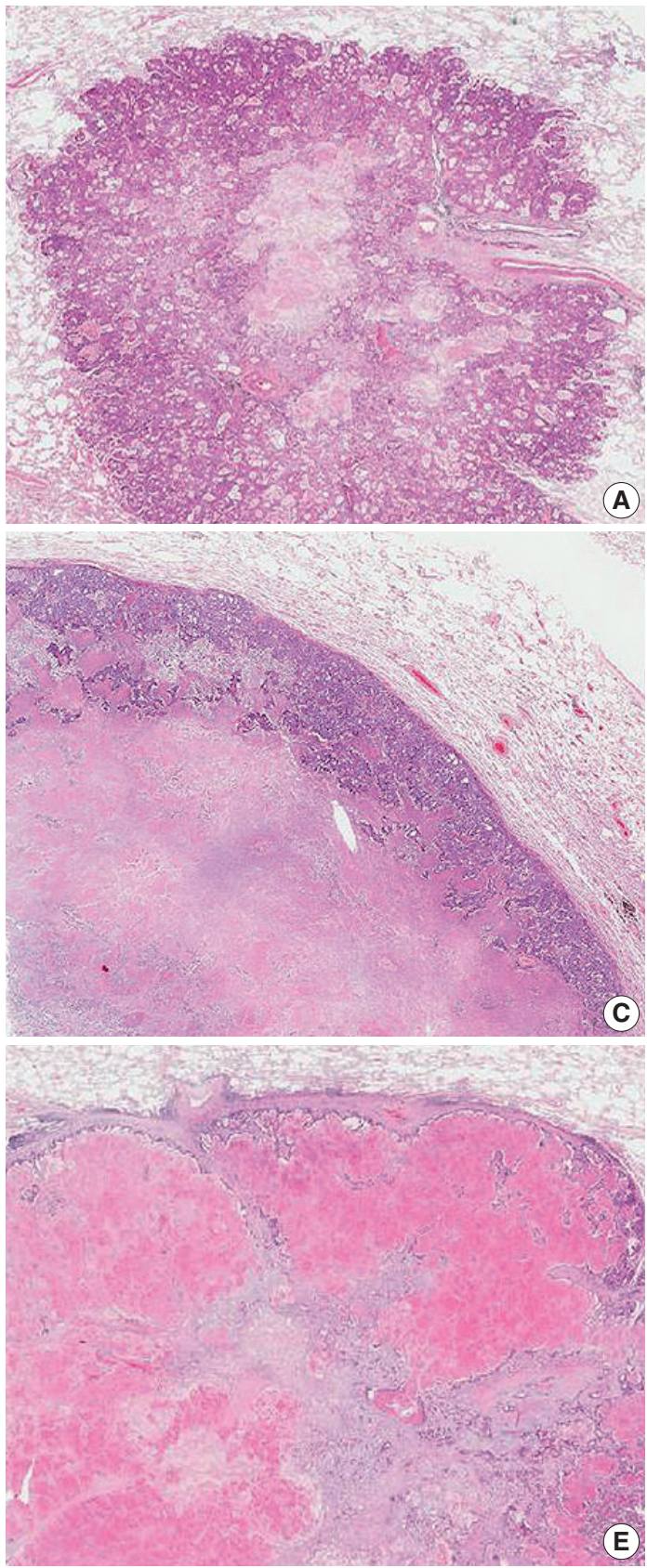

are summarized in Table 1 . The patient population included 57 $(62.0 \%)$ men and $35(38.0 \%)$ women with a mean age of 60.8 years (range, 31 to 83 years). The diameter of the largest CLOM ranged from 0.4 to $7.0 \mathrm{~cm}$, with a mean size of $1.8 \mathrm{~cm}$. The histology of the primary tumor included 86 well- or moderatelydifferentiated adenocarcinomas $(93.5 \%)$ and six poorly differen-
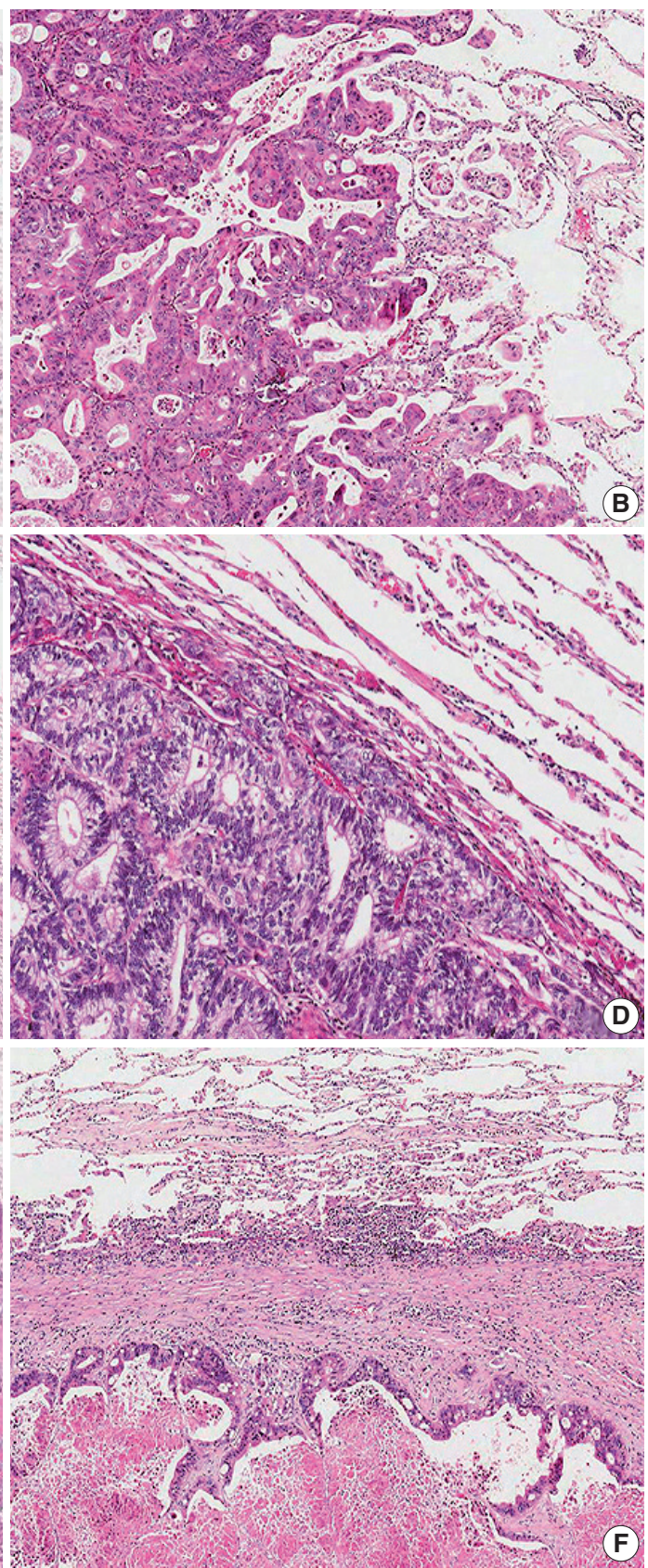

Fig. 1. Representative histologic features of the three different growth patterns of lung oligometastasis from colorectal adenocarcinoma. (A, B) In the aerogenous pattern, tumor clusters, nests, or single tumor cells spread into the air spaces of the lung parenchyma surrounding the edge of the tumor without destruction of the lung architecture, desmoplastic stroma, or inflammatory infiltrate. (C, D) In the pushing pattern, the metastasis grows by compression of the lung parenchyma, running parallel to the tumor-lung interface without desmoplastic stroma, and with only a mild inflammatory infiltrate. (E, F) In the desmoplastic pattern, the metastatic tumor is separated from the lung parenchyma by a desmoplastic rim infiltrated with lymphocytes. There is no direct contact between the tumor cells and lung parenchyma. 
Table 1. Relationship between histological growth patterns of colorectal lung oligometastasis and clinicopathological characteristics

\begin{tabular}{|c|c|c|c|c|c|}
\hline \multirow{2}{*}{ Clinicopathological characteristic } & \multicolumn{4}{|c|}{ Growth pattern } & \multirow{2}{*}{ p-value } \\
\hline & Aerogenous $(n=27)$ & Pushing $(n=32)$ & Desmoplastic $(n=6)$ & Mixed $(n=27)$ & \\
\hline \multicolumn{6}{|l|}{ Age at CLOM (yr) } \\
\hline Mean \pm SD & $57.63 \pm 11.32$ & $61.25 \pm 10.52$ & $69.50 \pm 11.50$ & $61.78 \pm 11.30$ & $.110^{\mathrm{a}}$ \\
\hline \multicolumn{6}{|l|}{ Sex } \\
\hline Male & $16(59.3)$ & $20(62.5)$ & $3(50.0)$ & $18(66.7)$ & $.872^{\mathrm{b}}$ \\
\hline Female & $11(40.7)$ & $12(37.5)$ & $3(50.0)$ & $9(33.3)$ & \\
\hline \multicolumn{6}{|l|}{ Size of CLOM (cm) } \\
\hline Mean \pm SD & $1.49 \pm 0.54$ & $2.03 \pm 1.40$ & $2.68 \pm 1.78$ & $1.66 \pm 0.59$ & $.033^{\mathrm{a}}$ \\
\hline \multicolumn{6}{|l|}{ No. of CLOMs } \\
\hline Single & $15(55.6)$ & $19(59.4)$ & $2(33.3)$ & $18(66.7)$ & $.491^{\mathrm{b}}$ \\
\hline Multiple (2 to $\leq 5)$ & $12(44.4)$ & $13(40.6)$ & $4(66.7)$ & $9(33.3)$ & \\
\hline \multicolumn{6}{|l|}{ Detection timing of CLOM } \\
\hline Synchronous & $8(29.6)$ & $9(28.1)$ & 0 & $7(25.9)$ & $.501^{\mathrm{b}}$ \\
\hline Metachronous & $19(70.4)$ & $23(71.9)$ & $6(100)$ & $20(74.1)$ & \\
\hline \multicolumn{6}{|l|}{ Frequency of CLOM } \\
\hline One time & $24(88.9)$ & $28(87.5)$ & $6(100)$ & $22(81.5)$ & $.635^{\mathrm{b}}$ \\
\hline More than one time & $3(11.1)$ & $4(12.5)$ & 0 & $5(18.5)$ & \\
\hline \multicolumn{6}{|l|}{ Location of $\mathrm{CRC}$} \\
\hline Colon & $11(40.7)$ & $14(43.8)$ & $3(50.0)$ & $9(33.3)$ & $.182^{b}$ \\
\hline Rectosigmoid & $1(3.7)$ & $8(25.0)$ & 0 & $7(25.9)$ & \\
\hline Rectum & $15(55.6)$ & $10(31.3)$ & $3(50.0)$ & $11(40.7)$ & \\
\hline \multicolumn{6}{|l|}{ Differentiation grade of CRC } \\
\hline Well & $15(55.6)$ & $21(65.6)$ & $2(33.3)$ & $12(44.4)$ & $.570^{\mathrm{b}}$ \\
\hline Moderately & $11(40.7)$ & $9(28.1)$ & $3(50.0)$ & $13(48.2)$ & \\
\hline Poorly & $1(3.7)$ & $2(6.3)$ & $1(16.7)$ & $2(7.4)$ & \\
\hline \multicolumn{6}{|l|}{ T category of CRC } \\
\hline $\mathrm{T} 1$ & 0 & $1(3.1)$ & 0 & $2(7.4)$ & $.566^{\mathrm{b}}$ \\
\hline T2 & $1(3.7)$ & $2(6.3)$ & $1(16.7)$ & $3(11.1)$ & \\
\hline T3 & $22(81.5)$ & $25(78.1)$ & $4(66.7)$ & $15(55.6)$ & \\
\hline T4 & $3(11.1)$ & $2(6.3)$ & $1(16.7)$ & $5(18.5)$ & \\
\hline Unknown & $1(3.7)$ & $2(6.3)$ & 0 & $2(7.4)$ & \\
\hline \multicolumn{6}{|l|}{$\mathrm{N}$ category of $\mathrm{CRC}$} \\
\hline NO & $8(29.6)$ & $14(43.8)$ & $1(16.7)$ & $11(40.7)$ & $.617^{\mathrm{b}}$ \\
\hline N1 & $8(29.6)$ & $8(25.0)$ & $3(50.0)$ & $9(33.3)$ & \\
\hline N2 & $10(37.0)$ & $8(25.0)$ & $2(33.3)$ & $5(18.5)$ & \\
\hline Unknown & $1(3.7)$ & $2(6.3)$ & 0 & $2(7.4)$ & \\
\hline
\end{tabular}

Values are presented as number (\%) unless otherwise indicated.

CLOM, colorectal lung oligometastasis; SD, standard deviation; CRC, colorectal cancer.

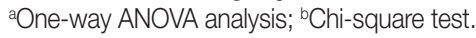

tiated adenocarcinomas (6.5\%). Fifty-four (58.7\%) patients had a single CLOM, while 38 ( $41.3 \%$ ) had multiple ( 2 to $\leq 5$ metastases) CLOMs. Twenty-four patients (26.1\%) had synchronous CLOM, and 68 (73.9\%) patients had metachronous CLOM. Further, $42.4 \%$ of our cases were located in the rectum, $83.7 \%$ had a locally advanced tumor (stage T3 or higher), and $57.6 \%$ demonstrated lymph node metastasis at the time of diagnosis.

\section{Histological growth patterns}

Overall, the aerogenous pattern was found in 29.4\% ( $\mathrm{n}=27)$ of the CLOMs, the pushing pattern was present in $34.7 \%$ ( $\mathrm{n}=$
32) of the CLOMs, the desmoplastic pattern was identified in 6.5\% ( $\mathrm{n}=6$ ) of the CLOMs, and the mixed pattern was found in $29.4 \%$ ( $n=27$ ) of the CLOMs (Table 1). Among the CLOMs with a mixed pattern, the pushing pattern (63.0\%) was the most predominant, and a combination of the pushing and aerogenous patterns (48.2\%) was the most common mixed pattern. Only one metastasis displayed all three growth patterns.

\section{Relationships between growth patterns and clinicopathological characteristics}

The size of the aerogenous pattern $(1.49 \pm 0.54 \mathrm{~cm})$ was sig- 
nificantly smaller than that of metastases with the pushing pattern $(2.03 \pm 1.40 \mathrm{~cm})$ or the desmoplastic $(2.68 \pm 1.78 \mathrm{~cm})$ pattern $(\mathrm{p}=$ .033). The number of CLOMs (single vs. multiple) per patient was not different among the growth patterns $(\mathrm{p}>.05)$. The detection timing (synchronous vs metachronous) and frequency (one time vs. more than once) of CLOM also did not show any significant difference among the growth patterns (each $\mathrm{p}>.05$ ). Although there was no significant correlation between the location of CRC and the growth pattern, the aerogenous pattern seemed to occur more frequently in the rectum than the nonaerogenous pattern, and this difference was marginally significant $(\mathrm{p}=.058)$. The differentiation grade and invasion depth of the primary tumor (T category of $\mathrm{CRC}$ ) and primary tumor lymph node status ( $\mathrm{N}$ category of $\mathrm{CRC}$ ) were not significantly correlated with the growth pattern of CLOM (each p > .05).

\section{Survival analysis}

Of the 92 patients included, 23 (25.0\%) died during followup. Median survival was 61.0 months, and the median followup time was 52.1 months (range, 0.2 to 155 months). When matched against all four growth patterns, a Kaplan-Meier analysis demonstrated no significant differences in overall survival according to the four growth patterns calculated from the diagnosis of CLOM or CRC (data not shown). However, the difference was more significant when patients were divided into two groups, with one group including patients with metastases having an aerogenous pattern and the other group including those with metastases having a pushing, desmoplastic, or mixed pattern. Patients showing an aerogenous pattern appeared to have a poorer prognosis with a median survival time of 58 months compared to a median survival of 64 months in patients with a non-aerogenous pattern, which was calculated from the time of diagnosis of CLOM ( $\mathrm{p}=.044)$ (Fig. 2A). The 5-year survival rate calculated from the diagnosis of $\mathrm{CRC}$ tended to be lower in patients with CLOM having an aerogenous pattern than in those with CLOM having a non-aerogenous pattern; however, the difference was marginally significant $(\mathrm{p}=.051)$ (Fig. 2B). In the multivariate $\mathrm{Cox}$ analysis, the aerogenous pattern was associated with a significantly poorer survival compared to the non-aerogenous pattern (hazard ratio [HR], 3.122; 95\% confidence interval [CI], 1.196 to $8.145 ; \mathrm{p}=.020)$, as the aerogenous pattern appeared to be an independent prognostic factor for poor overall survival (Table 2). The Cox regression analysis also showed the effects of the specific T category of CRC (HR, 5.412; 95\% CI, 1.961 to 14.938; $\mathrm{p}=.001$ ) and the detection timing of CLOM (HR, 3.008; 95\% CI, 1.106 to $8.185 ; \mathrm{p}=.031$ ) on survival time (Table 2).

Table 2. Multivariate Cox regression analysis for overall survival

\begin{tabular}{lccc}
\hline \multirow{2}{*}{ Variable } & \multicolumn{3}{c}{ Cox regression } \\
\cline { 2 - 4 } & $\mathrm{HR}$ & \multicolumn{1}{c}{$95 \% \mathrm{Cl}$} & $\mathrm{p}$-value \\
\hline Growth pattern & & & \\
Aerogenous & 3.122 & $1.196-8.145$ & .020 \\
$\quad$ Non-aerogenous & 1 & & \\
T category of CRC & & & .001 \\
T3-T4 & 5.412 & $1.961-14.938$ & \\
T1-T2 & 1 & & .031 \\
Detection timing of CLOM & & & \\
$\quad$ Synchronous & 3.008 & $1.106-8.185$ & \\
$\quad$ Metachronous & 1 & &
\end{tabular}

$\mathrm{HR}$, hazard ratio; $\mathrm{Cl}$, confidence interval; CRC, colorectal cancer; CLOM, colorectal lung oligometastasis.
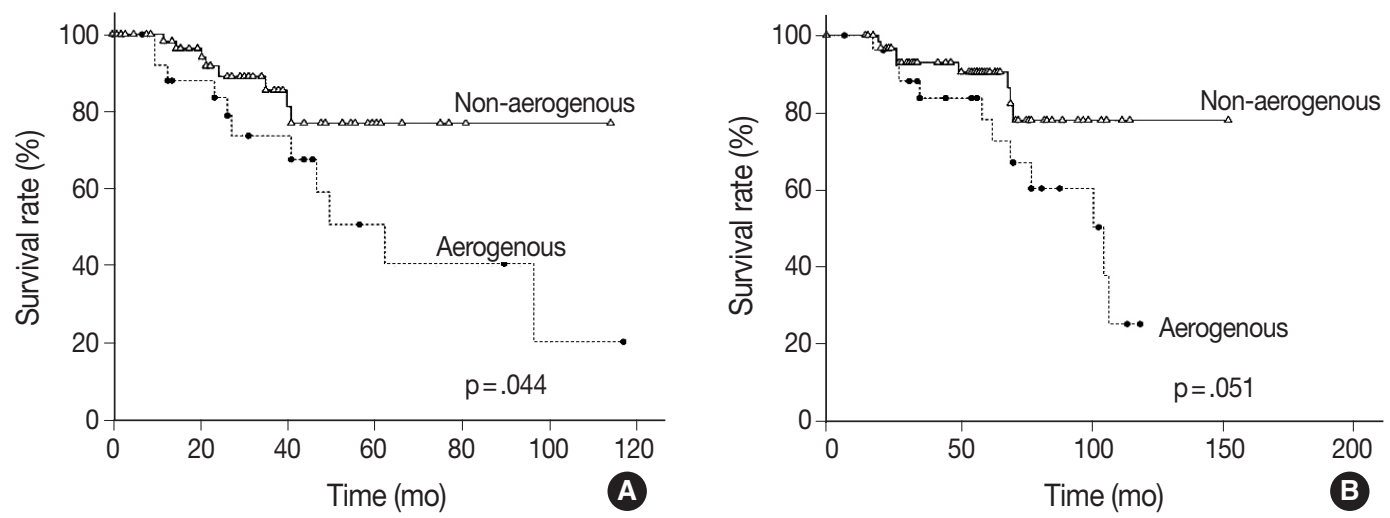

Fig. 2 . Kaplan-Meier curves illustrating the overall survival of patients with colorectal lung oligometastasis (CLOM) in relation to growth patterns. (A) The overall survival rate calculated from the diagnosis of CLOM shows a shorter survival time in patients with CLOM having an aerogenous pattern than in those with CLOM having a non-aerogenous pattern $(p=.044)$. (B) Although the difference was marginally significant $(p=.051)$, the overall survival rate calculated from the time of diagnosis of colorectal cancer tends to show a shorter survival length in patients with CLOM having an aerogenous pattern than in those with CLOM with a non-aerogenous pattern. 


\section{DISCUSSION}

In the present study, Kaplan-Meier and Cox regression analyses showed the prognostic significance of the histological growth patterns, as the aerogenous pattern seemed to be an independent prognostic factor for poor overall survival. This is one of few works to analyze the histopathological prognostic factors for classifying the potential of tumor aggressiveness in CLOM after pulmonary metastasectomy. If the present results can be reaffirmed, we recommend recoding the aerogenous pattern as a routine prognostic biomarker in the pathology report of CLOM.

The first possible mechanism behind these findings is explained by the new concept of spread through air spaces (STAS). Prior to the definition of STAS, one early study indicated that an aerogenous tumor spread and free floating cell clusters from metastatic CRC are unfavorable prognostic features. ${ }^{10}$ In a new statement regarding the invasiveness of primary lung adenocarcinoma, STAS have been recognized as an additional pattern of tumor invasiveness. Although the concept of STAS is still evolving, STAS consists of micropapillary clusters, solid nests or single cells beyond the edge of the tumor into the air spaces in the surrounding lung parenchyma. ${ }^{16}$ The loss of cell-to-cell adhesion or anchorage-independent growth of cancer cells may contribute to STAS. ${ }^{10}$ The presence of STAS in lung adenocarcinoma suggests a high risk of recurrence and invasion and is thus an important prognostic factor. ${ }^{17}$ We consider CLOM that has an aerogenous pattern to be comparable with STAS in primary lung cancer tumors. Although the definition of cancer infiltration into the surrounding lung parenchyma is different from that occurring in the liver, the aerogenous pattern is compatible with the replacement pattern of Vermeulen's procedure. ${ }^{11}$ The second possible explanation is that non-aerogenous metastases offer a benefit by incorporating the supporting architecture endogenous to the lung, as in colorectal liver metastasis. At the leading edge of CLOM, direct dynamic interaction between metastatic tumor and host cells, mediated by various kinds of cytokines, chemokines, or growth factors, may show tumor-promoting properties. ${ }^{12,18,19}$

Although our findings are interesting, there are several limitations to the present study. First, our study is retrospective, and it is limited by the fact that there was variation in the treatment protocol and modality before and after resection and also by the fact that responses to each treatment option were not evaluated in the survival analysis, possibly resulting in selection bias. Preoperative neoadjuvant therapy may also have influenced the morphology of growth patterns. However, we feel these limitations are outweighed based on Nielsen's opinion ${ }^{13}$ that the histologic findings of a growth pattern are really relevant, as the cohort reflects the actual patient status from a clinical point of view, although the survival data may not completely reflect the prognostic significance of growth patterns. Second, growth patterns were evaluated on the basis of only one tissue section per patient and included only the first metastasectomy, even for patients with multiple metastases that took place at different times. One study assessed the growth pattern of recurrent liver metastases and reported that $27.3 \%$ of recurrent metastases showed a different growth pattern than the first metastasis, while 31.8\% of recurrent metastases had a partly new growth pattern. ${ }^{13}$ Therefore, the growth pattern does not appear to be a host-specific feature. ${ }^{20}$ As the metastasis advances, the morphology of the tumor-lung interface can change either with the accumulation of desmoplastic stroma or with STAS when the speed of tumor growth exceeds the spread rate at the invasive front, which has been similarly reported in the liver. ${ }^{13,21}$ Third, a mixed-type was found in $29.4 \%$ of cases, and the pushing growth pattern was predominant (63.0\% of these cases). Moreover, $48.2 \%$ of these cases showed a combination of pushing and aerogenous growth patterns. Regarding the idea of STAS, the quantity of the aerogenous pattern might also be an important prognostic parameter. ${ }^{22}$ However, we could not demonstrate the intermediate survival rates for patients with CLOM having a mixed pattern, which reflects a heterogeneous tumor with both an aggressive aerogenous pattern and other more indolent patterns. Moreover, the case numbers of the combination subgroups of mixed pattern were too small for statistical evaluation to determine the prognostic effect according to the constituent parts of growth pattern. Another limitation of our study was that the number of patients showing each growth pattern was small, especially the desmoplastic subgroup, as previously discussed; therefore, this study may have been underpowered to detect some differences.

Despite these limitations, this study is the first to evaluate the histology-based prognostic factors for classifying the potential of tumor aggressiveness in the setting of pulmonary metastasectomy for CLOM. However, the current findings must be reproduced, and further investigation is needed to determine the clinical implications and the biological mechanism of growth patterns of CLOM in a well-adjusted study. A concomitant study of diverse metastatic mechanisms, including the complicated interaction with the lung microenvironment, might be helpful to understand the significance of histologic growth patterns of CLOM. 


\section{Conflicts of Interest}

No potential conflict of interest relevant to this article was reported.

\section{Acknowledgments}

This work was supported by the Dong-A University Research Fund.

\section{REFERENCES}

1. Weichselbaum RR, Hellman S. Oligometastases revisited. Nat Rev Clin Oncol 2011; 8: 378-82.

2. Corbin KS, Hellman S, Weichselbaum RR. Extracranial oligometastases: a subset of metastases curable with stereotactic radiotherapy. J Clin Oncol 2013; 31: 1384-90.

3. Comito T, Cozzi L, Clerici E, et al. Stereotactic ablative radiotherapy (SABR) in inoperable oligometastatic disease from colorectal cancer: a safe and effective approach. BMC Cancer 2014; 14: 619.

4. Inoue M, Ohta M, Iuchi K, et al. Benefits of surgery for patients with pulmonary metastases from colorectal carcinoma. Ann Thorac Surg 2004; 78: 238-44.

5. Rama N, Monteiro A, Bernardo JE, Eugénio L, Antunes MJ. Lung metastases from colorectal cancer: surgical resection and prognostic factors. Eur J Cardiothorac Surg 2009; 35: 444-9.

6. Pfannschmidt J, Dienemann H, Hoffmann H. Surgical resection of pulmonary metastases from colorectal cancer; a systemic review of published series. Ann Thorac Surg 2007; 84: 324-38.

7. Mitry E, Guiu B, Cosconea S, Jooste V, Faivre J, Bouvier AM. Epidemiology, management and prognosis of colorectal cancer with lung metastases: a 30-year population-based study. Gut 2010; 59: 1383-8.

8. Lee KS, Nam SK, Koh J, et al. Stromal expression of microRNA-21 in advanced colorectal cancer patients with distant metastases. J Pathol Transl Med 2016; 50: 270-7.

9. Iizasa T, Suzuki M, Yoshida S, et al. Prediction of prognosis and surgical indications for pulmonary metastasectomy from colorectal cancer. Ann Thorac Surg 2006; 82: 254-60.

10. Shiono S, Ishii G, Nagai K, et al. Histopathologic prognostic factors in resected colorectal lung metastases. Ann Thorac Surg 2005; 79:
278-82.

11. Vermeulen PB, Colpaert C, Salgado R, et al. Liver metastases from colorectal adenocarcinomas grow in three patterns with different angiogenesis and desmoplasia. J Pathol 2001; 195: 336-42.

12. Van den Eynden GG, Bird NC, Majeed AW, Van Laere S, Dirix LY, Vermeulen PB. The histological growth pattern of colorectal cancer liver metastases has prognostic value. Clin Exp Metastasis 2012; 29: 541-9.

13. Nielsen K, Rolff HC, Eefsen RL, Vainer B. The morphological growth patterns of colorectal liver metastases are prognostic for overall survival. Mod Pathol 2014; 27: 1641-8.

14. Nagashima I, Oka T, Hamada C, Naruse K, Osada T, Muto T. Histopathological prognostic factors influencing long-term prognosis after surgical resection for hepatic metastases from colorectal cancer. Am J Gastroenterol 1999; 94: 739-43.

15. Lunevicius R, Nakanishi $H$, Ito $S$, et al. Clinicopathological significance of fibrotic capsule formation around liver metastasis from colorectal cancer. J Cancer Res Clin Oncol 2001; 127: 193-9.

16. Travis WD, Brambilla E, Burke AP, Marx A, Nicholson AG. WHO classification of tumours of the lung, pleura, thymus and heart. 4th ed. Lyon: IARC Press, 2015.

17. Dai C, Xie H, Su H, et al. Tumor spread through air spaces affects the recurrence and overall survival in patients with lung adenocarcinoma > 2 to $3 \mathrm{~cm}$. J Thorac Oncol 2017; 12: 1052-60.

18. Paku S. Current concepts of tumor-induced angiogenesis. Pathol Oncol Res 1998; 4: 62-75.

19. Ueno H, Konishi T, Ishikawa Y, et al. Histological categorization of fibrotic cancer stroma in the primary tumor is an independent prognostic index in resectable colorectal liver metastasis. Am J Surg Pathol 2014; 38: 1380-6.

20. Eefsen RL, Van den Eynden GG, Høyer-Hansen G, et al. Histopathological growth pattern, proteolysis and angiogenesis in chemonaive patients resected for multiple colorectal liver metastases. J Oncol 2012; 2012: 907971.

21. Terayama N, Terada T, Nakanuma Y. Histologic growth patterns of metastatic carcinomas of the liver. Jpn J Clin Oncol 1996; 26: 24-9.

22. Warth A. Spread through air spaces (STAS): a comprehensive update. Transl Lung Cancer Res 2017; 6: 501-7. 